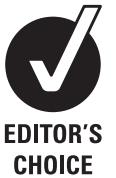

CHOICE
See ALS and FTD Special Edition, p 404

${ }^{1}$ Neuroscience Research Australia and University of New South Wales, Sydney, Australia. Australia

${ }^{2}$ Department of Neurology, Medical College, Trivandrum, Kerala, India

${ }^{3}$ School of Psychology, Edinburgh University, Edinburgh, UK

Correspondence to Professor John R Hodges, Neurosciences Research Australia, Barker Street, Randwick, New South Wales 2031, Australia;

j.hodges@neura.edu.au

Received 1 July 2011

Revised 24 August 2011

Accepted 6 September 2011

Published Online First

21 October 2011

\title{
Diagnostic criteria for corticobasal syndrome: a comparative study
}

\author{
Robert Mathew, ${ }^{1,2}$ Thomas H Bak, ${ }^{3}$ John R Hodges ${ }^{1}$
}

\section{ABSTRACT}

Introduction There are no well-established criteria for patients with corticobasal syndrome. The authors have attempted to clarify this area by comparing and applying three sets of well-known criteria (from Toronto, the Mayo Clinic and Cambridge).

Patient and methods The authors first compared the three criteria for overlap and differences, and then applied them to a group of 40 consecutive patients (22 men, mean age 67 years) with focal cortical syndrome characterised by apraxia and Parkinsonism, at both the early stages and later in their illness.

Results Despite an overall similarity, there were major differences in the criteria which affect their applicability. Cognitive impairment was ubiquitous even at presentation, with speech and language impairment the commonest feature. Some classic features, alien limb and myoclonus, were present in a minority only even late in their course. The three criteria were equally applicable to patients with advanced disease (Toronto $92.5 \%$, Cambridge $90 \%$ and Mayo $87.5 \%$ ). Thirty patients (75\%) satisfied all three criteria. Using this group as a 'gold standard', $73.3 \%$ fulfilled the Cambridge criteria at presentation compared with $46.7 \%$ and $36.7 \%$ for the Toronto and Mayo Clinic criteria, respectively. Concordance between criteria was poor.

Conclusion Cognitive impairment, especially language impairment, was prominent from onset of disease. The Cambridge criteria apply to a higher proportion of cases at an early stage of corticobasal syndrome. The authors suggest a minor modification to capture the high prevalence of aphasia.

\section{INTRODUCTION}

In the 40 years since its first description, corticobasal ganglionic degeneration (CBD) has become a topic of considerable interest. Yet, despite many reports, there are no agreed diagnostic criteria. As first described, $\mathrm{CBD}$ was considered a primary motor disorder characterised by asymmetrical rigidity with apraxia and variable other features, including cortical sensory loss, alien limb behaviour, myoclonus and dystonia. ${ }^{1}$ Cognitive abilities were stated to be relatively preserved. ${ }^{2}$ In fact, early diagnostic criteria stated that early dementia was an exclusion criterion. ${ }^{3}{ }^{4}$ Subsequent studies, however, described an increasing number of cases with cognitive deficits, appearing early in the disease and sometimes even before the onset of motor symptoms. ${ }^{5-16}$ As a result, CBD is now regarded as a complex disorder which affects motor and cognitive function, although the relative importance of these two major features remains controversial.
Neuropathologicaly patients with CBD were reported to have disorder with tau-positive intracellular inclusions, cortical ballooned neurons, frontoparietal neuronal loss and gliosis, and nigral and basal ganglia degeneration. ${ }^{9}$ More recently, it has become clear that this classic picture is present in a proportion of cases only, and many have other pathologies, most notably Alzheimer's disease (AD) and progressive supranuclear palsy. ${ }^{7} 13$ 17-22 Moreover, the classic neuropathology of $\mathrm{CBD}$ is found in patients who presented with progressive aphasia or frontotemporal dementia, making it difficult to maintain the term $\mathrm{CBD}$ as a unified clinicopathological entity. ${ }^{12} 142324$ This variability in pathology has led a number of authors to propose the label Corticobasal syndrome (CBS) for the clinical syndrome. ${ }^{14}$

A major confound which presents difficulty in the interpretation of the literature on CBS is the lack of agreed and validated diagnostic criteria. We surveyed current criteria; a number are poorly specified or have been used to a limited degree. $^{425-27}$ Three sets of criteria are more clearly defined: those proposed by Lang and Bergeron (Toronto), ${ }^{3}$ Boeve et al (Mayo clinic), ${ }^{28}$ and Bak and Hodges (Cambridge). ${ }^{13}{ }^{29}$ In an attempt to rationalise clinical criteria, we compared these three sets of criteria to examine areas of overlap and differences. We applied the three sets to a large group of patients with a clinical diagnosis of CBS, where the majority of the patients had focal cortical syndrome, apraxia and extrapyramidal motor features, seen in a clinic for patients with complex movement disorders, to test their applicability and relative performance, at both the early stages and later in the course. Our final aim was to see if any modifications to the criteria would better capture the syndrome of CBS.

\section{PATIENTS}

We searched the database of the Disorders of Movement and Cognition (DMC) Clinic, at Addenbrooke's Hospital, Cambridge for patients who received a label of CBS. Of 69 potential cases, 40 had comprehensive records and had been followed regularly. The DMC was established in 1997 as a tertiary multidisciplinary referral clinic for patients with complex movement disorders. In addition to the patients with CBS, the clinic also assessed 276 with progressive supranuclear palsy, 22 with frontotemporal dementia with motor neuron disease and 40 with multiple system atrophy. Patients with a purely cognitive presentation were assessed in the parallel Early Onset Dementia Clinic, 
also established in 1997. All patients were assessed by one of two experienced neurologists (JRH and THB). All underwent neurological examination, neuroimaging (CT or MRI) and neuropsychological assessments, including the Addenbrooke's Cognitive Examination $(\mathrm{ACE})^{3031}$ and were followed up whenever possible until death. During neurological examination, attention was paid to frontal executive functions, speech and language, limb praxis, visuospatial processing, bradykinesia, rigidity, involuntary movements and cortical sensory loss. Histopathological diagnosis was available in 16 patients: six patients had classic tau-positive CBD, eight Alzheimer's dementia, one FTD-U and one a necrotising leucoencephalopathy. The details of several patients have been published. ${ }^{10} 121332$

\section{DATA COLLECTION}

The clinical, neuroimaging information and neuropathology results were reviewed by a neurologist (RM), who was not involved with their diagnosis. The three selected clinical criteria (Toronto, Mayo Clinic and Cambridge criteria) were applied. For the purpose of this analysis, a feature was regarded as present if it appeared at any stage in the clinical course. A feature, which was not recorded, was considered as absent. Definitions of the clinical features extracted are shown in box 1.

\section{STATISTICS}

Agreement between pairs of criteria was calculated with the use of $\kappa$ statistics, which indicates the level of agreement, in which 0 reflects no agreement (ie, chance level) and 1 perfect agreement. Fisher's exact test was used to calculate differences across groups of patients. The statistics was calculated using SPSS V.18.

\section{RESULTS}

Comparison of three sets of criteria for the diagnosis of CBD Table 1 compares the three criteria. The features common to all criteria included extrapyramidal signs (rigidity, dystonia) and cortical motor features (limb apraxia, alien limb phenomenon, myoclonus and cortical sensory loss). There were, however, significant differences. First, the Toronto criteria give no importance to the temporal profile of illness, while others include onset and progression of illness. Second, cognitive features and extrapyramidal features are given equal weight in the Cambridge criteria. The Mayo criteria include cognitive dysfunction, but secondary to the movement disorder. The third difference is the presence of exclusion features in the Toronto criteria including progressive supranuclear palsy (vertical gaze palsy), multiple system atrophy (severe autonomic disturbance), idiopathic Parkinson's disease (rest tremor, responsiveness to levodopa), Alzheimer's dementia (early dementia) and structural lesions (lesions on imaging). Fourth, none of the criteria specify the stage at which given features are expected to be present, with uncertainty, therefore, as to whether any given clinical manifestation is expected early or late, in the course of the disease.

In addition to these major differences, minor differences across sets are present. Even the seemingly similar categories are complicated by qualification terms. For example, rigidity, one of the features common to all criteria, is described as simply 'rigidity,' 'focal rigidity' and 'akinetic rigid syndrome.' Likewise myoclonus is also described as 'reflex focal myoclonus' by two criteria and by just 'myoclonus' by the Cambridge criteria. When the categories are applied in the strict sense, only two features, alien limb and cortical sensory loss, remain common to all the three criteria. In our analysis, we combined features which are clearly very similar (eg, myoclonus, rigidity).

\section{Box 1 Definition of clinical features}

Asymmetry

- Asymmetry in motor features (rigidity, bradykinesia, myoclonus or dystonia) or cortical sensory motor symptoms (apraxia, alien-limb syndrome or cortical sensory loss)

- Akinetic rigidity

- Rigidity and bradykinesia were both present

- Asymmetrical rigidity

- A difference of at least one grade across the sides when the rigidity was graded according to a four-point scale

- Gait instability

- Postural instability on retropulsion test or gait apraxia

- Limb apraxia

- The inability to carry out learnt, skilled motor acts with limbs despite preserved motor and sensory systems, coordination, comprehension and cooperation, incorporating deficits copying meaningless gestures (limb kinetic) or meaningful gestures to command (ideomotor); inability to perform the Luria three-step test was not considered significant evidence of apraxia

- Alien-limb syndrome

- Apparently purposeful actions of one hand against the patient's intention ${ }^{33}$

- Speech and language impairment

- The presence of a significant motor speech disorder with distorted articulation or aphasia, typically non-fluent

- Frontal dysfunction

- Decreased verbal fluency, concreteness in thinking, impaired reasoning, slowed information processing, lack of insight, poor information retrieval, and impaired execution of sequential actions. Personality changes may include apathy, reflecting a lack of concern about personal behaviour or the behaviour of others or disinhibition. ${ }^{34}$

- Frontal release signs

- At least one of the following was present: grasp, groping, snout, pout, or palmo-mental reflex ${ }^{35}$

- Myoclonus

- Included reflex myoclonus, focal myoclonus, asymmetrical myoclonus or reflex focal myoclonus.

\section{Patient demographics}

The male:female ratio was $22: 18$, and the mean age at presentation was 67 years (SD 7.34). The mean duration of illness at the time of presentation was 3 (SD 2.1) years, and the mean duration of follow-up was 4.9 (SD 2) years. Death occurred at a mean duration of 4.9 (SD 2.7) years from last visit.

Of the 40 patients, CBS was the primary diagnosis at their first visit in $25(62.5 \%)$. Three were diagnosed as having Alzheimer's dementia, one with progressive supranuclear palsy, one with frontotemporal dementia and one with progressive non-fluent aphasia. CBS was the primary diagnosis of all the cases by the time of their last visit.

Clinical features at initial presentation and on final assessment All the patients had an insidious onset and gradual progression. None of the patients showed a significant response to levodopa. The commonest features were disturbance of speech and language in 38 (95\%) followed by limb apraxia $30(75 \%)$. Akinetic rigidity was present in $24(60 \%)$. Of the 38 patients with speech and language impairment, all were aphasic, of 
Table 1 Comparison of three criteria

\begin{tabular}{|c|c|c|c|}
\hline Features & Toronto criteria & Mayo criteria & Cambridge criteria \\
\hline \multicolumn{4}{|l|}{ Temporal profile and treatment response } \\
\hline Insidious onset & 0 & $1+$ & 0 \\
\hline Asymmetrical onset & 0 & 0 & $1+$ \\
\hline Chronic progressive course & 0 & $1+$ & 0 \\
\hline Insidious onset and chronic progression & 0 & 0 & $1-$ \\
\hline Levodopa-resistant & 0 & 1 & 1 \\
\hline Sustained levodopa response & Ex & 0 & 0 \\
\hline \multicolumn{4}{|l|}{ Motor features } \\
\hline Rigidity & 1 & 0 & 0 \\
\hline Focal rigidity & 0 & 1 & 0 \\
\hline Asymmetrical rigidity & 1 & 1 & 0 \\
\hline Akinetic rigid syndrome & 0 & 0 & $1+$ \\
\hline Dystonia & 0 & 1 & 1 \\
\hline Focal dystonia & 1 & 0 & 0 \\
\hline Myoclonus & 0 & 0 & 1 \\
\hline Reflex focal myoclonus & 1 & 1 & 0 \\
\hline Focal or asymmetrical myoclonus & 0 & 1 & 0 \\
\hline Rest tremor & Ex & 0 & 0 \\
\hline \multicolumn{4}{|l|}{ Cortical sensory motor symptoms } \\
\hline Apraxia & 1 & 0 & $1+$ \\
\hline Asymmetric/focal apraxia & 0 & 1 & 0 \\
\hline Number processing deficit & 0 & 0 & 1 \\
\hline Cortical sensory loss & 1 & 1 & 1 \\
\hline Alien limb & 1 & 1 & 1 \\
\hline Visual or sensory hemineglect & 0 & 1 & 0 \\
\hline \multicolumn{4}{|l|}{ Cognitive symptoms } \\
\hline Early dementia & Ex & 0 & 0 \\
\hline Frontal dysfunction & 0 & $1-$ & $1+$ \\
\hline Visuospatial deficit & 0 & 0 & 1 \\
\hline Apraxia of speech/nonfluent aphasia & 0 & 1 & 1 \\
\hline \multicolumn{4}{|l|}{ Exclusion features } \\
\hline Early vertical gaze palsy & Ex & 0 & 0 \\
\hline Severe autonomic disturbance & Ex & 0 & 0 \\
\hline Imaging of other pathology & Ex & 0 & 0 \\
\hline Imaging features & 0 & $1-^{*}$ & 0 \\
\hline No identifiable cause & 0 & $1+$ & 0 \\
\hline
\end{tabular}

which the majority $(60 \%)$ had a non-fluent syndrome, and most of the remaining $40 \%$ were anomic. Less than half (17) also had dysarthria. A sizeable number, 22 (55\%), also had dysgraphia. The various clinical features were divided into three broad categories (see table 2). Cognitive impairment was seen in all patients $(100 \%)$ followed by cortical sensory motor symptoms in $31(77.5 \%)$ and primary motor symptoms in $27(67.5 \%)$. Cognitive involvement alone was present in three (7.5\%). In 24 $(60 \%)$, features from all the three domains were seen at their first visit.

At the final visit to the clinic (see table 2) the commonest features were still speech and language impairment seen in 38 , followed by apraxia 37 (92.5\%) and frontal-lobe dysfunction 30 (75\%). Myoclonus was present in almost half, and the alien-limb phenomenon was seen in $40 \%$.

\section{Applicability of diagnostic criteria}

At presentation to the clinic, the greatest number fulfilled the Cambridge criteria (67.5\%) followed by the Toronto $(47.5 \%)$, while the Mayo criteria (32.5\%) were the least sensitive. The difference between the former and the latter two was significant $(p=0.005)$. Only 30\% satisfied all three criteria, and $27.5 \%$ did not qualify for any criteria on first visit. Overall, almost threequarters $(72.5 \%)$ satisfied one of the three sets of criteria, while almost half $(47.5 \%)$ satisfied any of the two criteria. Of the patients with histopathological diagnosis, five patients each with $\mathrm{CBD}$ and $\mathrm{AD}$ were diagnosed by the Cambridge criteria on first visit.

After the final visit, the proportion of patients fulfilling the three criteria was very similar; Toronto $92.5 \%$, Cambridge $90 \%$ and Mayo $87.5 \%$. Thirty patients (75\%) satisfied all the three diagnostic criteria, while all except one patient satisfied at least two criteria. All six patients with histopathologically proven tau-positive $\mathrm{CBD}$ as well as the eight patients with $\mathrm{AD}$ pathology found their place in the group satisfying all the three criteria. The patient with FTD-U, however, did not meet all the three criteria.

Given the differences between the criteria, we took as a 'gold standard' for clinical diagnosis the 30 patients who eventually satisfied all three criteria and then examined the accuracy of diagnosis at presentation. The Cambridge criteria diagnosed the maximum number of patients on first visit (73.3\%) followed by the Toronto criteria $(46.7 \%)$, while the Mayo criteria were the least applicable (36.7\%). 
Table 2 Percentage of patients having various manifestations at presentation and final visit

\begin{tabular}{lll}
\hline & $\begin{array}{l}\text { First visit-no of } \\
\text { patients (\%) }\end{array}$ & $\begin{array}{l}\text { Final visit- no of } \\
\text { patients (\%) }\end{array}$ \\
\hline Motor features & $27(67.5)$ & $39(97.5)$ \\
Akinetic rigidity & $24(60)$ & $29(72.5)$ \\
Gait instability & $15(37.5)$ & $25(62.5)$ \\
Myoclonus & $12(30)$ & $18(45)$ \\
Cortical motor sensory features & $31(77.5)$ & $37(92.5)$ \\
Limb apraxia & $30(75)$ & $37(92.5)$ \\
Alien-limb syndrome & $11(27.5)$ & $16(40)$ \\
Cortical sensory loss & $08(20)$ & $18(45)$ \\
Cognitive features & $40(100)$ & $40(100)$ \\
Speech and language & $38(95)$ & $38(95)$ \\
Frontal dysfunction & $23(57.5)$ & $30(75)$ \\
Visuospatial deficits & $19(47.5)$ & $22(55)$ \\
Frontal release signs & $13(32.5)$ & $24(60)$ \\
\hline
\end{tabular}

Table 3 shows concordance and weighted $\kappa$ among various criteria. Agreement was mild or poor between all pairs

We explored any modifications of the Cambridge criteria that would improve its applicability, replacing frontal executive dysfunction with language impairment as a major criterion. The modified Cambridge criteria are shown in box 2 .

\section{DISCUSSION}

This is the first study to examine the utility of various criteria for the diagnosis of CBS. ${ }^{325}$ We compared three well-known sets of criteria and found that although they share key similarities, there were important differences. When applied to a group of 40 patients, seen in a specialist clinic, there was poor concordance. All three criteria could be applied equally well in the later stages, but in the earlier stages the Cambridge criteria had significantly wider applicability, almost certainly due to the weight given to cognitive and language dysfunction.

The three clinical criteria chosen are all consensus criteria derived on the basis of clinical experience by experts in the field, rather than being derived from prospective natural history studies with clinico-pathological correlation. The differences reflect, therefore, differences in clinical experience and interest of the teams involved, while there were clear similarities and overlap, particularly in the need for an insidious onset and progressive course (except in Toronto criteria) and the presence of (usually) asymmetrical akinetic rigid syndrome with limb apraxia. There were other key differences notably in the emphasis on, or omission of, cognitive dysfunction from the criteria. In the Toronto criteria, the presence of early dementia was an exclusion criterion in sharp contrast to Cambridge criteria where cognitive dysfunction formed one of the important groups of diagnostic features.

Some motor features that are regarded as characteristic of CBS, notably myoclonus and alien-limb phenomenon, were present in less than a half of patients even when last seen in the

Table 3 Concordance and $\kappa$ values between various criteria

\begin{tabular}{lll}
\hline Criteria & Percentage concordance & К values \\
\hline Mayo and Cambridge & 82 & 0.125 \\
Mayo and Toronto & 84 & 0.172 \\
Cambridge and Toronto & 87 & 0.219 \\
\hline
\end{tabular}

\section{Box 2 Modified Bak and Hodges criteria} (Cambridge criteria) $\uparrow$

- Mandatory criteria

- Insidious onset and gradual progression

- No sustained response to levodopa treatment $\dagger$

- Major and minor criteria*

- Motor features

Akinetic rigid syndrome

Focal or segmental myoclonus

Asymmetrical dystonia

- Cortical motor sensory features

Limb apraxia

Alien limb phenomenon

Cortical sensory loss or dyscalculia

- Cognitive features

Speech and language impairment $\ddagger$

Frontal executive dysfunction§

Visuospatial deficits

${ }^{*}$ Criteria in italics are major criteria, and the rest are minor criteria.

tThe response of the parkinsonism to levodopa therapy should be tested with at least $25 / 250 \mathrm{mg}$ of carbidopa/levodopa administered three times a day for at least 2 months. The response to levodopa is considered poor when the extrapyramidal features fail to show marked improvement, or the therapeutic effect is transient (ie, lasts less than a year). ${ }^{34}$

¥Includes aphasia, dysarthria and dysgraphia.

§Includes frontal release signs reduced verbal fluency and other abnormal tests of frontal functions.

TFor a diagnosis of CBS, the patient should satisfy all mandatory criteria, two major criteria (in italics) and two minor criteria.

clinic, and were considerably rarer at presentation. These features may have been overemphasised in description of CBS and feature too prominently in some criteria-for example, Toronto criteria.

It is notable that all 40 patients in our study had some form of cognitive impairment, even at the time of presentation, manifest as language impairment, memory loss or frontal-lobe dysfunction. It is now generally accepted that cognitive impairment is common in CBS; however, the exact prevalence remains unknown, as the vast majority of studies have been based on a small number of patients. Also, the interpretation of the available estimates is hampered by methodological issues. ${ }^{11}$ Prior studies have shown that impairment of frontal-lobe function was one of the most consistent cognitive abnormalities. It has been estimated that executive dysfunction with behavioural disturbance or poor performance on frontal-lobe tests is present in at least $50 \%$ of the patients with CBD. ${ }^{15}$ Dubois and colleagues showed that a group of 21 patients were all impaired on frontal-lobe function. ${ }^{36}$ Our study, in keeping with Dubois et al, found a large number of patients with cognitive impairment at presentation.

One striking observation was the prominence of speech and language abnormalities, which were present in the vast majority of patients, even at their first assessment. A similar observation was made by Graham et $a l^{10}$ in a smaller study, which found that the majority of their patients had evidence of aphasia, but this was typically subclinical and apparent only after detailed testing of speech and language function. Moreover, all the 
patients in the Graham et al study had limb apraxia or extrapyramidal symptoms at the time of presentation. Their review of the literature revealed that over a third of reported CBS cases had clinically obvious aphasia most often of the non-fluent type. ${ }^{10}$ Murray et al also reported language dysfunction to be the most commonly reported complaint early in the CBD with word-finding difficulty, effortful speech or dysgraphia. ${ }^{37}$ The present study goes further. Nearly all patients had speech and language impairment at presentation that was characterised as non-fluent in type. Apraxia of speech has been associated with underlying $\tau$ pathology. ${ }^{38-40}$ Unfortunately, we are not able to say what proportion of our patients had apraxia of speech since a detailed perceptual analysis of speech samples was not available. This finding adds to the growing awareness of the clinical overlap between CBS and progressive non-fluent aphasia. ${ }^{14}$

The main reason for the difference between the Cambridge, Toronto and Mayo criteria is the equal emphasis in the Cambridge criteria on motor and cognitive features together with their use of major versus minor criteria. We have suggested a slight modification while keeping the basic structure whereby frontal executive dysfunction, which was a major criterion, was replaced by speech and language impairment. For better specificity, some of the criteria (insidious onset and gradual progression, and levodopa insensitivity) were made mandatory.

Diagnostic criteria for any disorder should ideally be validated against an independent biological 'gold standard.' Unfortunately, the variable pathology in CBS presents insuperable problems in this regard. As highlighted in the introduction, a proportion of patients have classic $\tau$-positive intracellular inclusions with cortical ballooned neurons, ${ }^{9}$ but a considerable minority have other pathologies, most notably $\mathrm{AD} .^{713}{ }^{18-21}$ As a surrogate for this ideal validation, we took the subgroup of patients who fulfilled all of three established diagnostic criteria comprising 30 (75\%) of the 40 cases. All six patients with histopathological proven tau-positive CBD were included in this core group as well as the eight patients with $\mathrm{AD}$. It has previously been suggested that a number of clinical features, notably early memory complaints and deficits on the ACE, and a lack of behavioural changes are indicative of $\mathrm{AD}$ pathology, but we were unable to support or refute this prior finding. ${ }^{13}$

The large number of patients in this cohort with cognitive features at presentation raises the issues related to referral bias. Even though such a possibility cannot be ruled out totally, the influence of such a bias is likely to be low. The DMC clinic was established to evaluate patients with disorders of movement and cognition. Thus, virtually all patients had motor features, even if these were not predominant. Only three patients had cognitive features alone at presentation. A parallel clinic in Cambridge was established in 1997 in which large numbers of patients with pure cognitive disorders were assessed. We would argue that the high rate of language and cognitive dysfunction reflects, instead, the interest and expertise of the two senior neurologists ( $\mathrm{TB}$ and JRH) who evaluated all of the patients and the use of a systematic evaluation tools designed to uncover and classify disorders of cognition which is not the situation in most movement disorder clinics.

In conclusion, the available clinical criteria for CBS differ considerably. All three apply equally well to patients in the advanced stages of the disorder, but at presentation the Cambridge criteria appear to capture a higher proportion of cases because of their more structured nature and their emphasis on cognitive dysfunction. Although the number of cases with postmortem pathology is relatively small, there were no major differences between those with classic CBD and those with
AD. ${ }^{14}$ Prospective longitudinal studies with clinico-pathological correlation are clearly required.

Acknowledgements We thank M Hornberger and E Mioshi for statistical and database help.

Funding JRH is supported by an Australian Research Council Federation Fellowship (FF\# 0776229).

\section{Competing interests None.}

Contributors RM: research project conception, statistical analysis and drafting of manuscript; THB: research project conception and critique of manuscript; JRH: research project conception and design, review of manuscript.

Provenance and peer review Not commissioned; externally peer reviewed.

\section{REFERENCES}

1. Rebeiz JJ, Kolodny EH, Richardson EP Jr. Corticodentatonigral degeneration with neuronal achromasia. Arch Neurol 1968;18:20-33.

2. Rinne J0, Lee MS, Thompson PD, et al. Corticobasal degeneration. A clinical study of 36 cases. Brain 1994;117:1183-96.

3. Lang $\mathrm{AE}$, Riley DE, Bergeron C. Cortico-basal ganglionic degeneration. In: Calne DB, ed. Neurodegenerative Diseases. Philadelphia: WB Saunders, 1994:877-94.

4. Kumar R, Bergeron C, Pollanen MS, et al. Cortical basal ganglionic degeneration. In: Jankovic J, Tolosa E, eds. Parkinson's Disease and Movement Disorders. Baltimore: Williams \& Wilkins, 1998:297-316.

5. Bergeron C, Pollanen MS, Weyer L, et al. Unusual clinical presentations of corticalbasal ganglionic degeneration. Ann Neurol 1996;40:893-900.

6. Soliveri P, Monza D, Paridi D, et al. Cognitive and magnetic resonance imaging aspects of corticobasal degeneration and progressive supranuclear palsy. Neurology 1999;53:502-7.

7. Grimes DA, Lang AE, Bergeron CB. Dementia as the most common presentation of cortical-basal ganglionic degeneration. Neurology 1999;53:1969-74.

8. Feany MB, Mattiace LA, Dickson DW. Neuropathologic overlap of progressive supranuclear palsy, Pick's disease and corticobasal degeneration. J Neuropathol Exp Neurol 1996;55:53-67.

9. Schneider JA, Watts RL, Gearing $M$, et al. Corticobasal degeneration: neuropathologic and clinical heterogeneity. Neurology 1997;48:959-69.

10. Graham NL, Bak T, Patterson K, et al. Language function and dysfunction incorticobasal degeneration. Neurology 2003;61:493-9.

11. Graham NL, Bak TH, Hodges JR. Corticobasal degeneration as a cognitive disorder Mov Disord 2003;18:1224-32

12. Mathuranath PS, Xuereb JH, Bak T, et al. Corticobasal ganglionic degeneration and/ or frontotemporal dementia? A report of two overlap cases and review of literature. J Neurol Neurosurg Psychiatry 2000;68:304-12.

13. Shelley BP, Hodges JR, Kipps CM, et al. Is the pathology of corticobasal syndrome predictable in life? Mov Disord 2009:24:1593-9.

14. Kertesz A, Martinez-Lage P, Davidson W, et al. The corticobasal degeneration syndrome overlaps progressive aphasia and frontotemporal dementia. Neurology 2000;55:1368-75.

15. Kertesz A, McMonagle P. Behavior and cognition in corticobasal degeneration and progressive supranuclear palsy. J Neurol Sci 2010:289:138-43.

16. Lantos PL. Diagnostic criteria for corticobasal degeneration. J Neurol Neurosurg Psychiatry 2000;69:705-6.

17. Feany MB, Dickson DW. Neurodegenerative disorders with extensive tau pathology: a comparative study and review. Ann Neurol 1996;40:139-48.

18. Tsuchiya K, Ikeda K, Uchihara T, et al. Distribution of cerebral cortical lesions in corticobasal degeneration: a clinicopathological study of five autopsy cases in Japan. Acta Neuropathol 1997:94:416-24.

19. Chand P, Grafman J, Dickson D, et al. Alzheimer's disease presenting as corticobasal syndrome. Mov Disord 2006;21:2018-22.

20. Vanvoorst WA, Greenaway MC, Boeve BF, et al. Neuropsychological findings in clinically atypical autopsy confirmed corticobasal degeneration and progressive supranuclear palsy. Parkinsonism Relat Disord 2008;14:376-8.

21. Boeve BF. Links between frontotemporal lobar degeneration, corticobasa degeneration, progressive supranuclear palsy, and amyotrophic lateral sclerosis. Alzheimer Dis Assoc Disord 2007;21:S31-8.

22. Ling H, O'Sullivan SS, Holton JL, et al. Does corticobasal degeneration exist? A clinicopathological re-evaluation. Brain 2010;133:2045-57.

23. Lippa CF, Cohen R, Smith TW, et al. Primary progressive aphasia with focal neuronal achromasia. Neurology 1991:41:882-6.

24. Arima K, Uesugi H, Fujita I, et al. Corticonigral degeneration with neuronal achromasia presenting with primary progressive aphasia: ultrastructural and immunocytochemical studies. J Neurol Sci 1994;127:186-97.

25. Riley DE, Lang AE, Lewis A, et al. Cortical-basal ganglionic degeneration. Neurology 1990:40:1203-12.

26. Watts RL, Brewer RP, Schneider JA, et al. Corticobasal degeneration. In: Watts RL, Koller WC, eds. Movement Disorders: Neurologic Principles and Practice. New York: McGraw-Hill,1997:611-12

27. Halpern C, Clark R, Moore P, et al. Verbal mediation of number knowledge: evidence from semantic dementia and corticobasal degeneration. Brain Cogn 2004;56:107-15. 
28. Boeve BF, Lang AE, Litvan I. Corticobasal degeneration and its relationship to progressive supranuclear palsy and frontotemporal dementia. Ann Neurol 2003;54(Suppl 5):S15-19.

29. Bak TH, Hodges JR, Thomas HB. Corticobasal degeneration: clinical aspects. In: Duyckaerts C, Litvan I, eds. Handbook of Clinical Neurology. Vol. 89. Amsterdam: Elsevier, 2008:509-21.

30. Mathuranath PS, Nestor PJ, Berrios GE, et al. A brief cognitive test battery to differentiate Alzheimer's disease and frontotemporal dementia. Neurology 2000:55:1613-20.

31. Mioshi E, Dawson K, Mitchell J, et al. The Addenbrooke's Cognitive Examination Revised (ACE-R): a brief cognitive test battery for dementia screening. Int J Geriatr Psychiatry 2006:21:1078-85.

32. Bak TH, Crawford LM, Hearn VC, et al. Subcortical dementia revisited: similarities and differences in cognitive function between progressive supranuclear palsy (PSP), corticobasal degeneration (CBD) and multiple system atrophy (MSA). Neurocase 2005;11:268-73.

33. Goldberg G, Mayer NH, Toglia JU. Medial frontal cortex infarction and the alien hand sign. Arch Neurol 1981;38:683-6.
34. Litvan I, Agid Y, Calne D, et al. Clinical research criteria for the diagnosis of progressive supranuclear palsy (Steele-Richardson-Olszewski syndrome): report of the NINDS-SPSP international workshop. Neurology 1996;47:1-9.

35. Neary D, Snowden JS, Gustafson L, et al. Frontotemporal lobar degeneration: a consensus on clinical diagnostic criteria. Neurology 1998;51:1546-54.

36. Dubois B, Slachevsky A, Litvan I, et al. The FAB: a Frontal Assessment Battery at bedside. Neurology 2000;55:1621-6.

37. Murray R, Neumann M, Forman MS, et al. Cognitive and motor assessment in autopsy-proven corticobasal degeneration. Neurology 2007:68:1274-83.

38. Josephs KA, Boeve BF, Duffy JR, et al. Atypical progressive supranuclear palsy underlying progressive apraxia of speech and nonfluent aphasia. Neurocase 2005:11:283-96.

39. Josephs KA, Duffy JR. Apraxia of speech and nonfluent aphasia: a new clinical marker for corticobasal degeneration and progressive supranuclear palsy. Curr Opin Neurol 2008;21:688-92.

40. Josephs KA, Duffy JR, Strand EA, et al. Clinicopathological and imaging correlates of progressive aphasia and apraxia of speech. Brain 2006:129 1385-98.

\section{Have confidence in your decision making.}
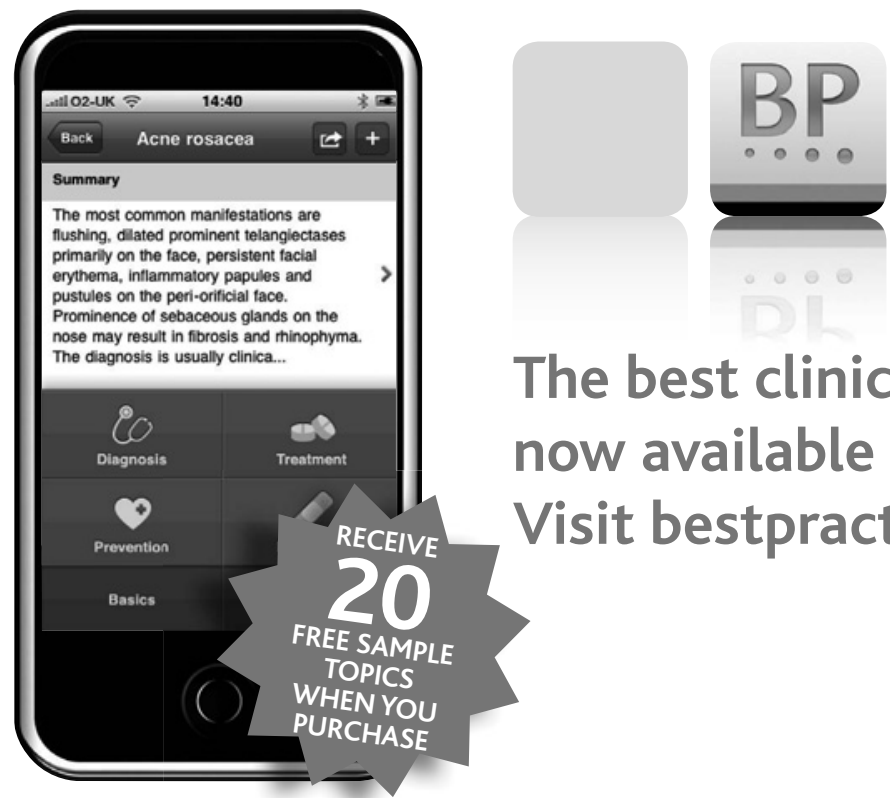

The best clinical decision support tool is now available as an app for your iPhone. Visit bestpractice.bmj.com/app

\section{clinicians $\bullet$ medical students $\bullet$ nurses $\bullet$ healthcare practitioners}

